\title{
New Developments in Human Embryonic Research Put Pressure on the 14-Day Rule
}

\section{Robert Ungard}

Medical Science Program, McMaster University.

Email: ungardr@mcmaster.ca

Since 1979, the "14-day rule" has stood as a regulatory and legal limit on in vitro human embryo growth. It stipulates that human embryos cannot be maintained in vitro past 14 days of development. The limit was first proposed following the ethical discussion surrounding the first in vitro human fertilization in 1970 at the University of Cambridge (1). Since then, it has been adopted by many countries, either as scientific guidelines or set into legislation, as has been done in Canada. Recent developments in human embryonic research are beginning to test that rule for the first time, both by the maintenance of embryos up to 13 days for the first time, and by the creation of embryo-like structures, to which the 14-day rule may not apply.

The 14-day barrier was selected as that is the point of human development at which germ layer differentiation begins and the primitive streak - the early site of gastrulation and the site that later becomes the spine - becomes apparent. At this point an embryo is a blastocyst, barely visible to the naked eye. Before 14 days, approximately $50 \%$ of embryos at this stage are sloughed off by the uterus - a high attrition rate - and embryos can fuse together or split into twins, indicating that the embryo may not yet be a discernible individual. Once the primitive streak develops at 14 days, the embryo is considered an individual, as it can no longer fuse with another embryo or split into twins.

The 14-day rule sought to strike a balance between the need to ascribe value to human embryos without abolishing the scientific investigation of viable human embryos. It is also just one of many regulations governing human embryonic and genetic research. Nonetheless, the 14-day rule is not without critique, both from those who believe that it places too little value on the moral status of an embryo, and from those who believe it is an arbitrary obstacle to important human developmental research.

Until last year, the rule existed without any practical methodological challenge. It was only in 2016 that two scientific groups in the United States and the United Kingdom attained the capacity to grow self-organizing human embryos for 13 days in vitro - for the first time coming up against the barrier of the 14-day limit $(2,3)$. Never before had researchers been able to extend their in vitro embryonic growth further. In addition, human embryonic stem cells were recently induced to develop features characteristic of later developmental stages, including primitive streaks and distinct germ cell layers; however, those were not intact whole embryos, and as such, did not violate the 14-day rule (4).

In addition to this immediate methodological challenge to the 14-day limit, more taxing ethical and legal challenges will soon emerge. Early this year, a report on the implications of synthetic human entities with embryo-like features (SHEEFs) cast doubt on the ability of the simple 14-day rule to effectively direct research utilizing these or similar structures (5). SHEEFs are not a new concept, but they will soon become a new reality. They are embryo-like assemblies of cells created from pluripotent human stem cells. These could potentially be viable embryo-like cell clusters created from induced pluripotent stem cells, or they could be structures distinct from embryos, but displaying similar features of development (4).

Adult human cells have yet to be induced to form a SHEEF; however, this work has progressed further with mouse cells, where researchers have maintained combinations of cultured embryonic and trophoblast stem cells up to 6.5 days with comparable development to maternally-developing embryos (6). In addition, research with human pluripotent stem cells induced from adult fibroblasts and grown in vitro has led to the development of organoids, including livers shown to be functional upon implantation and growth in animal models (7).

The applicability of the 14-day rule to SHEEFs depends on the definition of "embryo." Is a "gastruloid" created from human embryonic stem cells that exhibits three germ layers but no other familiar structural pat- 
terning an embryo? Is a structure apparently indistinguishable from a human embryo but created with human pluripotent stem cells induced from adult tissue also an embryo, and as such, subject to the same structural protections?

This technology is clearly in its infancy; however, the implications are that these current embryo-like cell clusters could one day be sustained in vitro - or in vivo in the case of induced cells - to a point of development where more complex human features would develop. These complex human features, like a nervous or circulatory system, offer great potential for scientific investigation. However, the 14-day rule was designed to protect a biological individual from experimentation, and since these features are distinctly human, this may spark ethical controversy.

Recently, several groups have spoken out in favour of amending or extending the 14-day rule, both to accommodate the progress of human embryonic research and to account for the difficulty of defining SHEEFs $(5,8)$. To some it seems absurd to have allowed the 14-day rule to stand, only for the rule to possibly be broken by researchers (9). Without the 14-day rule, what would stand as a new limit? A modified 14-day rule would need to take into account the new practical reality of growing human embryos beyond 14 days. It would also be valuable to include direction for research into SHEEFs and other structures that have similarities but do not conform to the structural definition of a human embryo.

It is inevitable that novel discoveries, as well as ethical, social, and legal challenges, will soon arise from research into viable human embryos in vitro and SHEEFs. It would be prudent for these concerns to be addressed shortly - before researchers advance beyond the limits and imagination of current ethical frameworks.
2. Deglincerti A, Croft GF, Pietila LN, Zernicka-Goetz M, Siggia ED, Brivanlou AH. Self-organization of the in vitro attached human embryo. Nature. 2016;533(7602):251-254.

3. Shahbazi MN, Jedrusik A, Vuoristo S, Recher G, Hupalowska A, Bolton V, et al. Self-organization of the human embryo in the absence of maternal tissues. Nat Cell Biol. 2016;18(6):700-708.

4. Warmflash A, Sorre B, Etoc F, Siggia ED, Brivanlou AH. A method to recapitulate early embryonic spatial patterning in human embryonic stem cells. Nat Methods. 2014;11(8):847-854.

5. Aach J, Lunshof J, lyer E, Church GM. Addressing the ethical issues raised by synthetic human entities with embryo-like features. Elife. 2017;6.

6. Harrison SE, Sozen B, Christodoulou N, Kyprianou C, ZernickaGoetz M. Assembly of embryonic and extra-embryonic stem cells to mimic embryogenesis in vitro. Science. 2017;356(6334).

7. Takebe T, Sekine K, Enomura M, Koike H, Kimura M, Ogaeri T, et al. Vascularized and functional human liver from an iPSC-derived organ bud transplant. Nature. 2013;499(7459):481-484.

8. Hyun I, Wilkerson A, Johnston J. Embryology policy: revisit the 14day rule. Nature. 2016;533(7602):169-171.

9. Greely H. In vitro human embryos and the 14 day rule. Stanford Center for Law and the Biosciences Blog. 2016. Available from: https://law.stanford.edu/2016/05/04/in-vitro-human-embryosand-the-14-day-rule-2/ [Accessed 2017 Apr 12].

\section{References}

1. Zimmer C. A new form of stem-cell engineering raises ethical questions. New York Times. 2017. Available from: https://www. nytimes.com/2017/03/21/science/embryonic-stem-cells-synthetic-embryos-sheefs.html?_r=0 [Accessed 2017 Apr 12].

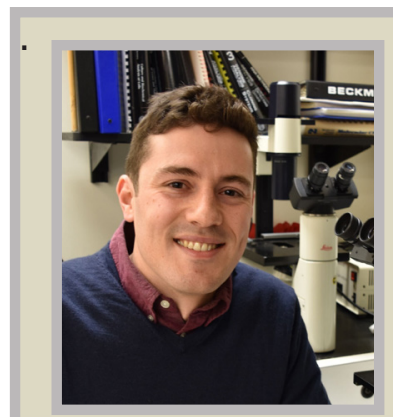

\section{Robert Ungard}

Robert is a PhD Candidate in the Medical Science program of McMaster University. Robert's research is focussed on pain - particularly cancer pain, and ways to reduce that pain that maintain patient quality of life throughout their treatment. 